\title{
Analysis of Thermal and Reaction Times for Hydrogen Reduction of Lunar Regolith
}

\author{
U. Hegde ${ }^{1}$, R. Balasubramaniam¹, and S. Gokoglu ${ }^{2}$ \\ ${ }^{1}$ National Center for Space Exploration Research, Cleveland, Ohio, 44135 \\ ${ }^{2}$ NASA John H Glenn Research Center, Cleveland, Ohio 44135 \\ ${ }^{1}$ (216) 433-8744, uday.hegde@grc.nasa.gov
}

\begin{abstract}
System analysis of oxygen production by hydrogen reduction of lunar regolith has shown the importance of the relative time scales for regolith heating and chemical reaction to overall performance. These values determine the sizing and power requirements of the system and also impact the number and operational phasing of reaction chambers. In this paper, a Nusselt number correlation analysis is performed to determine the heat transfer rates and regolith heat up times in a fluidized bed reactor heated by a central heating element (e.g., a resistively heated rod, or a solar concentrator heat pipe). A coupled chemical and transport model has also been developed for the chemical reduction of regolith by a continuous flow of hydrogen. The regolith conversion occurs on the surfaces of and within the regolith particles. Several important quantities are identified as a result of the above analyses. Reactor scale parameters include the void fraction (i.e., the fraction of the reactor volume not occupied by the regolith particles) and the residence time of hydrogen in the reactor. Particle scale quantities include the particle Reynolds number, the Archimedes number, and the time needed for hydrogen to diffuse into the pores of the regolith particles. The analysis is used to determine the heat up and reaction times and its application to NASA's oxygen production system modeling tool is noted.
\end{abstract}

Keywords: hydrogen reduction, ilmenite, lunar regolith, shrinking core.

PACS: 47.70.Fw, 82.40.-g.

\section{INTRODUCTION}

Hydrogen reduction of lunar regolith has been proposed as a viable technology for oxygen production on the moon. (Taylor and Carrier 1992, McKay and Allen 1996, Rice, Hermes and Musbah 1997). The reduction processes involve solid-gas reactions. Such systems are well developed for earth applications but mainly for the purpose of metal extraction as opposed to water vapor and oxygen production for lunar applications. In addition, in the lunar context, the reactor must interface with the other sub-system processes such as upstream regolith extraction and beneficiation, and downstream electrolysis and phase separation in a way that establishes the most favorable balance between efficiency, robustness, and equivalent system mass. For example, the reduction of ilmenite may be represented as:

$$
\mathrm{FeO} . \mathrm{TiO}_{2}+\mathrm{H}_{2} \Leftrightarrow \mathrm{Fe}+\mathrm{TiO}_{2}+\mathrm{H}_{2} \mathrm{O}
$$

It is carried out in the reactor sub-system. The formed water may be electrolyzed in the electrolyzer subsystem to produce oxygen and hydrogen.

From upmass and cost considerations, it is essential to operate these systems on the lunar surface in as nearly a closed-loop manner as possible for the hydrogen. It is in this context that knowledge of processing times in the various sub systems becomes important. For example, a mismatch between the flux of water exiting the reactor and the amount of water that may be processed by the electrolyzer must be mitigated by design such as sizing the appropriate buffer tank. This paper investigates the factors that influence the overall processing time in the reactor sub system.

The temperature of the reaction is one of the important parameters of the process. Ilmenite is just one constituent of mare basalt and it cannot be assumed that regolith beneficiation (e.g., in this case, removal of the non-ilmenite components) will be carried out prior to the reactor processes. The sintering and melting temperature of the unbeneficiated regolith is noted to be in the range of 1370-1670 K (Chambers 1995, McKay and Allen 1996). However, hydrogen reduction has been found to be effective also at lower temperatures, e.g., in the range of 970$1270 \mathrm{~K}$ (Yoshida et al., 2000). Therefore, while three-phase (gas-liquid-solid) reactors for hydrogen reduction may 
be applicable at post-melt temperatures, these are not considered here and attention is restricted to gas-solid interactions. In order to provide a basis for discussion, it is assumed that the chemical reduction of the regolith occurs in a gas-solid fluidized bed reactor.

The conversion process in the reactor consists of the following overall steps: (a) transfer of regolith into the reactor, (b) fluidization and heating of the regolith, (c) reaction of hydrogen with the ilmenite portion of the regolith, (d) outflow of left-over hydrogen and produced water vapor, and (e) removal of the spent regolith from the reactor. In this paper, attention is focused on steps (b) -(d).

The reported values for the thermal diffusivity, $\beta$, of lunar regolith are very low (Heiken, Vaniman and French 1991). In the absence of a gas flow, the time to heat the regolith bed over a distance $\delta$ is proportional the ratio $\delta^{2} / \beta$, which results in unacceptably large values. On the other hand, hydrogen has a relatively high thermal conductivity and helps to stir the bed so that having hydrogen flow through the bed decreases the heating time appreciably. Thus, it is advantageous to fluidize the bed during the regolith heating step. There are also advantages related to reducing regolith particle agglomeration and sintering which can adversely impact reaction times and water production rates.

The objectives of this paper are (a) to develop analytical models for regolith heating and for chemical reduction by hydrogen in order to identify parameters influencing the heating and reaction times, (b) to identify the parameters that govern these times, and (c) to obtain relationships for the reaction time in the two limits of diffusion (i.e., mass transfer) control and chemical equilibrium control. As noted earlier, these times are important from the overall oxygen production system perspective. The thermal analysis is based upon a Nusselt number correlation. The reaction model considered is based upon a "shrinking core" diffusion-reaction model for the gas-solid reduction process (Levenspiel 1998) which is coupled with the inflow/outflow of gases. The methodology has been discussed in our earlier work (Hegde, Balasubramaniam and Gokoglu 2007). It appears consistent with results of regolith particulate analysis conducted on Apollo-returned samples (McKay and Allen 1996) which suggest that the ilmenite particles are not completely solid but have some porosity that would enable gases to diffuse into the particles, i.e., the $\mathrm{FeO}$ portion can be essentially completely converted leaving behind the reduced iron.

In the next section, the regolith heating is considered first and a model for the heating time and required power is developed. Pertinent results from the shrinking core analysis are also presented. Subsequently, in the Results section, factors impacting these processes are evaluated and discussed and applications of the model to NASA's oxygen production system modeling tool are noted.

\section{MODEL DEVELOPMENT}

In the first sub-section below, the time taken to heat the regolith is calculated using a Nusselt number correlation for fluidized bed heat transfer. This is followed by a summary of the particle conversion analysis and the coupling between the particle conversion and the bulk reactor flow.

\section{Heat Transfer Analysis}

It is assumed that the regolith is heated by a cylindrical rod located centrally in the cylindrical reactor. The surface temperature of the heater rod can be controlled e.g., by adjusting the current through it as in an electrical heater or by controlling the energy flux as in a solar concentrator heat pipe. The different pathways for heat transfer in the bed are gas convection and conduction, particle motions, and possibly, radiative transfer.

Fluidized bed heat transfer correlations developed in the literature under convective-conductive conditions suggest that the controlling step is the heat transfer from the heater surface to the gas film next to it. Direct transfer of heat to the bed particles adjoining the heater is not efficient because the contact area between particles and the heater surface is small. If the bed is well stirred by fluidization, its temperature is nearly uniform. In such a case, a Nusselt number may be defined which relates the heat transfer to the bed through the gas film in contact with the heater, i.e., the regolith bed is taken to be a uniform entity. The reactor wall is not usually insulated from the bed, so in general, there will also be heat transfer from the bed to the reactor wall. If the reactor wall is not too thick and its thermal conductivity is high, the wall may be assumed to be at a uniform temperature. Furthermore, if the reactor's thermal mass (i.e., the product of its mass and specific heat) is small compared to that of the bed, it is reasonable to assume that the temperature of the reactor is the same as that of the bed. 
The heating rate with the above scenario is given by:

$$
M_{e f f} C_{e f f} \frac{d T}{d t}=h A_{e f f}\left(T_{h}-T\right),
$$

where the thin film heat transfer coefficient, h, is related to the Nusselt number, $\mathrm{Nu}$, by:

$$
N u=2 h r_{p} / \lambda .
$$

In Eq.(2), $\mathrm{M}_{\mathrm{eff}} \mathrm{C}_{\mathrm{eff}}$ is the effective value of the product of the mass and specific heat and is a weighted average of the values for the gas, regolith, and reactor. $A_{\text {eff }}$ is the effective or active surface area of the heater rod in contact with the bed.

The solution of Eq. (2) for the instantaneous bed temperature T is given by:

$$
T=T_{h}-\left(T_{h}-T_{1}\right) \exp \left(-h A_{e f f} t /\left(M_{e f f} C_{e f f}\right)\right) .
$$

Conversely, the time to raise the bed temperature from $T_{1}$, to a temperature $T_{2}$ is given by:

$$
t_{h}=-\frac{M_{e f f} C_{e f f}}{h A_{e f f}} \ln \left(\frac{T_{h}-T_{2}}{T_{h}-T_{1}}\right)
$$

Various fluidized bed heat transfer Nusselt number correlations are available in the literature. For example, the following one (Borodulya et. al. 1991) appears to describe the situation adequately:

$$
N u=0.74 A r^{0.1}\left(\frac{\rho_{s}}{\rho_{g}}\right)^{0.14}\left(\frac{C_{p s}}{C_{p g}}\right)^{0.24}(1-\varepsilon)^{2.3}+0.46 \operatorname{Re} \operatorname{Pr} \frac{(1-\varepsilon)^{2.3}}{\varepsilon} .
$$

The Archimedes number, Ar, the Reynolds number, Re, and the Prandtl number, Pr, are defined as follows:

$$
\begin{gathered}
A r=8 g r_{p}^{3} \rho_{g}\left(\rho_{s}-\rho_{g}\right) / \mu^{2}, \\
\operatorname{Re}=2 u r_{p} \rho_{g} / \mu, \\
\operatorname{Pr}=C_{p g} \mu / \lambda_{g} .
\end{gathered}
$$

The energy, $\mathrm{E}$, required to heat the bed and reactor from $\mathrm{T}_{1}$ to $\mathrm{T}_{2}$, in the absence of other losses (e.g., radiative), is given by:

$$
E=M_{e f f} C_{e f f}\left(T_{2}-T_{1}\right)
$$

An average power equired is obtained by dividing $\mathrm{E}$ by the time, $\mathrm{t}_{\mathrm{h}}$. Note that the instantaneous power, $\mathrm{M}_{\text {eff }} \mathrm{C}_{\text {eff }} \mathrm{DT} / \mathrm{dt}$, can be obtained by substituting the solution for T (Eq. (4 )) into Eq. (2).

Since radiative losses vary as the fourth power of temperature, they can become important as the reactor is heated to higher temperatures. It is expected that there would be insulation around the reactor that would reduce this loss. This loss can be accounted for by including it in the energy required. 


\section{Chemical conversion analysis}

The chemical conversion analysis couples the "shrinking core" model with the inflow and outflow of the gases and has been discussed in earlier work (Hegde, Balasubramaniam and Gokoglu, 2007). Here, some results are presented to highlight the findings from the model and to show how the characteristic times of the process are obtained.

Defining the conversion $\alpha$ by:

$$
\alpha=1-\frac{r_{s}^{3}}{r_{p}^{3}}
$$

the shrinking core analysis of a single particle yields a relationship between $\alpha$ and the associated conversion time, t:

$$
3[1-\alpha]^{2 / 3}-2[1-\alpha]-1=-\frac{6 D\left[c_{x}-c_{s}\right] t}{\rho_{a} r_{p}^{2}} .
$$

Further, assuming equilibrium conditions at the reaction surface, the above equation reduces to:

$$
3[1-\alpha]^{2 / 3}-2[1-\alpha]-1=-\frac{6 D k c_{0} t}{(1+k) \rho_{a} r_{p}^{2}} .
$$

Note that an expression for the equilibrium constant, $\mathrm{k}$, as a function of only the reaction temperature has been provided (William and Erstfeld, 1979):

$$
k=10^{(-2126.1 / T+0.6439)} .
$$

The time for complete conversion is given by setting $\alpha$ to unity.

\section{Coupling Particle Analysis with the Reactor Bulk Flow}

Assuming that the gas velocity, concentration of hydrogen and the associated conversion $\alpha$ are functions of axial distance along the reactor, and the inlet hydrogen concentration is constant in time, the following equation has been obtained:

$$
-4 \ln \left[\frac{1-(1-\alpha)^{1 / 3}}{1-\left(1-\alpha_{0}\right)^{1 / 3}}\right]+\ln \left[\frac{2(1-\alpha)^{1 / 3}+1}{2\left(1-\alpha_{0}\right)^{1 / 3}+1}\right]=3(1-\varepsilon) \frac{D}{r_{p}^{2} u} x,
$$

where $\alpha_{0}$ is the value of $\alpha$ at $x=0$ and is given by Eq. (13).

In certain cases, closed form solutions may be obtained for the concentration of hydrogen and extent of conversion in the reactor. An example is when the regolith and gases are sufficiently stirred or well-mixed so that gradients in conversion and gas concentration are smeared out. In this well-stirred limit, the concentration of hydrogen exiting the reactor is also the average concentration in the reactor and is given by:

$$
c(L)=\frac{c_{0}}{1+k}\left\{1+\frac{k}{\left[1+3(1-\varepsilon)\left(\frac{D}{r_{p}^{2}}\right) \frac{[1-\alpha]^{1 / 3}}{\left[1-(1-\alpha)^{1 / 3}\right]} \frac{L}{u}\right]}\right\}
$$




\section{RESULTS}

Regolith batch sizing will depend upon the oxygen production required and the processing time per batch. In general, the processing time per batch is the sum of the heating and chemical conversion times. This section considers factors influencing these times based upon the analysis presented earlier.

\section{Heating Time}

Equation (5) indicates that the heating time is related directly to the Nusselt number and batch size (i.e., regolith mass) and inversely related to the active surface area of the heater. The Nusselt number depends upon the Reynolds number of the gas flow relative to the regolith particles, the Archimedes number, and the Prandtl number of the fluidizing gas. The Prandtl number is practically constant with temperature. The Reynolds number is related to the fluidizing velocity and particle size. The particle size is fixed by the regolith characteristics and there may be some flexibility in this regard depending upon the preprocessing facilities available. The fluidizing velocity is also related to the particle size. The Archimedes number is mainly dependent upon the regolith density (which is much greater than the gas density). The most flexible way of changing the heating time seems to be by changing the regolith batch size, active heater area, and/or power input to the heater.

The active heater surface area may be increased by increasing the heater diameter. For a fixed reactor diameter, this also increases the height, $\mathrm{L}$, of the regolith bed which is also conducive to heat transfer. However, this may have implications for the reaction time as will be discussed later. The regolith batch size may be reduced to decrease the heating time but this may be restricted from overall oxygen production considerations.

The power input to the heater is related to the heater temperature $T_{h}$. From Eq. (5), it is clear that increasing the heater temperature will decrease the time to heat the regolith to a given temperature $T_{2}$. However, there are limitations to raising $T_{h}$ substantially over $T_{2}$ e.g., the presence of hot spots or regions in the regolith bed which are at higher than desired temperature.

\section{Chemical Time}

The shrinking core analysis provides an expression for the conversion time of a single particle. In many instances it is assumed that the conversion time of the regolith bed is the same as the single particle conversion time. Equation (15) provides a means for determining the conditions under which this holds. Choosing $\mathrm{x}=\mathrm{L}$ (i.e., the height of the regolith bed), it is clear that the right hand side of Eq. (15) is related to the ratio of the gas residence time in the bed, $\mathrm{L} / \mathrm{u}$, to its diffusion time in a particle, $\mathrm{r}_{\mathrm{p}}{ }^{2} / \mathrm{D}$. This time factor ratio plays an important role in determining the extent of conversion in the bed. For example, the upstream end (entrance region) of the bed is always exposed to fresh hydrogen and would be the first to get converted. The time ratio factor gives a measure of the conversion in the remaining portions of the bed. Figure 1 plots the conversion, $\alpha(L)$, at the downstream end (exit region) as a function of the time ratio factor for the case where $\alpha_{0}$ is equal to unity, i.e., when the particles at the entrance are already completely converted. As the time ratio factor becomes small (i.e., the intra-particle diffusion time is much greater than the gas residence time in the regolith bed) it can be seen from Fig. 1 that the conversion, $\alpha(\mathrm{L})$, also approaches unity, i.e., the particles at the downstream end of the bed (and more generally, at any location in the bed) are also consumed fully. This indicates that the time rate of chemical conversion for all particles in the bed is given by Eq. (13). The corresponding time for complete conversion may be termed the chemical time in the diffusion controlled limit. Setting $\alpha$ to one in Eq. (13) yields:

$$
t_{\text {DLimit }}=\frac{(1+k) \rho_{a} r_{p}^{2}}{6 D k c_{0}}
$$






FIGURE 1. Dependence of the Regolith Conversion at the Downstream End of the Reactor Bed Upon the Time Ratio Factor $3(1-\varepsilon) \mathrm{DL} / \mathrm{r}_{\mathrm{p}}^{2} \mathrm{u}$.

On the other hand, as the residence time becomes much larger compared to the diffusion time, i.e., the time ratio factor becomes large, it can be seen from Fig. 1 that the conversion occurs first primarily near the upstream end of the bed and not much conversion occurs downstream since $\alpha(\mathrm{L})$ is small for this condition. This is referred to as the chemical equilibrium limit, i.e., the residence time in the bed is long enough so that the water concentration reaches its chemical equilibrium value in the gas phase. At this point no further conversion takes place. In this limiting case, the chemical time may be estimated by assuming equilibrium concentration of water in the outflow. Then, the molar outflow of water is given by :

$$
\psi=\frac{k}{(1+k)} u A c_{0},
$$

and the total molar water yield is:

$$
M_{\text {total }}=\psi t_{\text {Re sLimit }} .
$$

Equating this to the available molar water yield in the regolith provides the chemical time in the residence time limit or equilibrium limit:

$$
t_{\text {ResLimit }}=\rho_{a}(1-\varepsilon) V_{\text {bed }} / \psi .
$$

Since the conversion time in the diffusion limit depends inherently upon the particle characteristics such as its size and the intra-particle gas diffusivity, it is clear that this is a lower limit to the conversion time. The equilibrium 
limiting conversion time is variable however, depending upon the flow velocity and bed height. Varying these quantities is a way to slow down the conversion rate. This may be useful from an overall system design perspective.

\section{Application to System Design}

The analysis of heating and chemical times presented here may be applied to sizing and design of the reactor assembly. It has been noted in earlier studies that the regolith reduction rate increases directly with the temperature, (McKay and Allen 1996) presumably due to increase in the effective diffusion coefficient (Hegde, Balasubramaniam and Gokoglu 2007). This implies that most of the conversion occurs at the operating temperature. In other words, the heating and chemical reduction are essentially serial operations for a given batch of regolith. Thus, a system comprising two reactors may be used for increased throughput. Ignoring for current purposes, the regolith loading and unloading times into the reactor chamber, it is clear that designing for equal heating and reaction times will ensure continuous production of oxygen, i.e., as one batch of regolith is being heated the other batch is being chemically reduced.

The equations provided here provide a framework for achieving the proper design parameters. The diffusion limited chemical time (Eq. (17)) provides the minimum time for conversion. Equations (5) and (10) in conjunction provide the power and heater rod characteristics (e.g., effective area) for a given heating time, e.g., the minimum reaction time. If the power available is less than the heating power required, the heating time has to be increased. In such a case, the reaction time needs to be modified e.g., by driving the reactor dimensions toward the residence limited conversion time (Eq. (20)). Clearly, this is an iterative process. Currently, NASA is developing an overall system model incorporating these ideas (Santiago-Maldonado 2007).

\section{CONCLUSIONS}

Analytical models for regolith heating and for chemical reduction by hydrogen have been developed to identify parameters influencing the heating and reaction times. These times are important from the overall oxygen production system perspective. The regolith heating model is based upon a Nusselt number correlation for fluidized bed heat transfer. While particle size and parameters such as the particle Reynolds and Archimedes numbers play a role in the heating time determination, it appears that the most feasible way of controlling this time is by adjusting the active heater surface area and mass of the regolith per batch. The chemical reduction model results from coupling a shrinking core type of analysis with the overall regolith bed flow characteristics. It is shown that the chemical time in the diffusion limit is also the lower limit for the conversion time. However, by adjusting the residence time of the gases in the regolith bed, the conversion time can be increased.

\section{NOMENCLATURE}

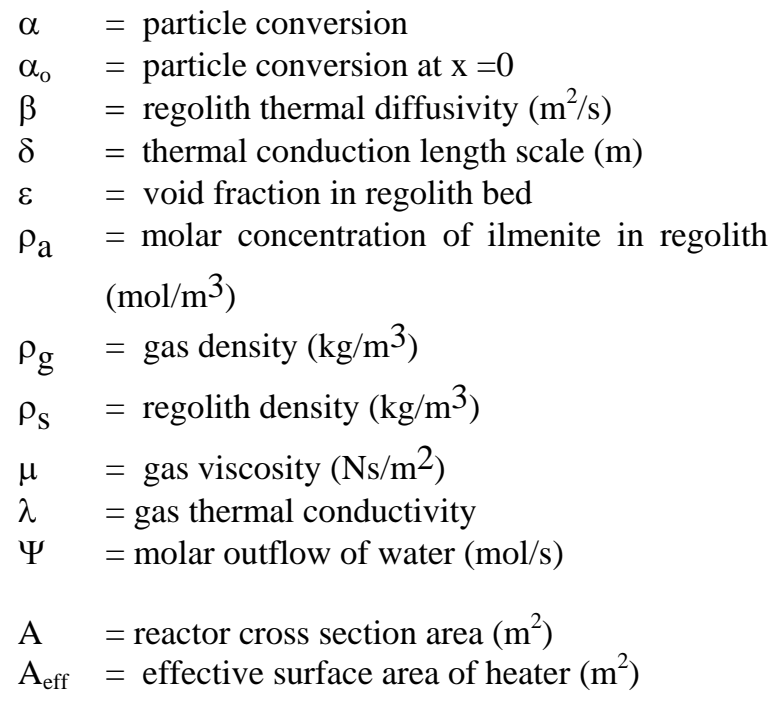

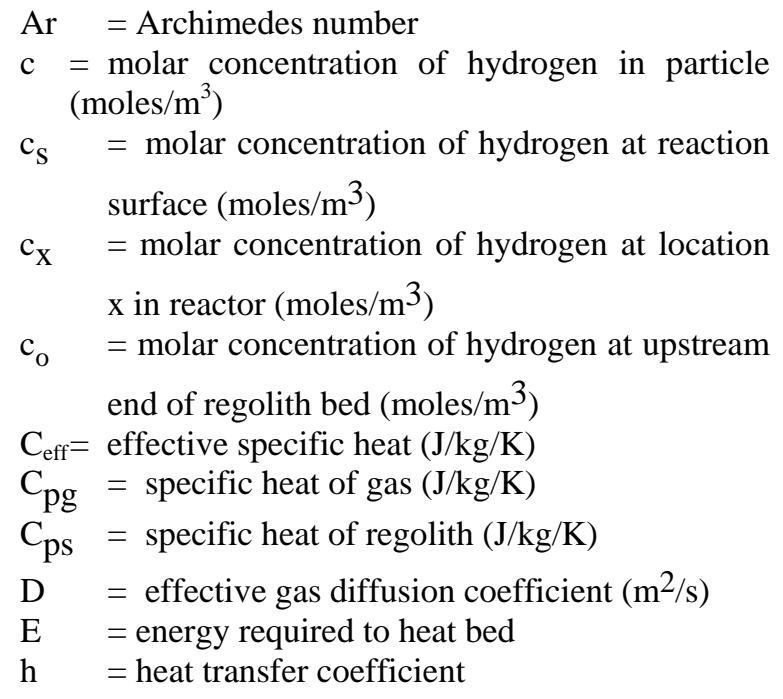



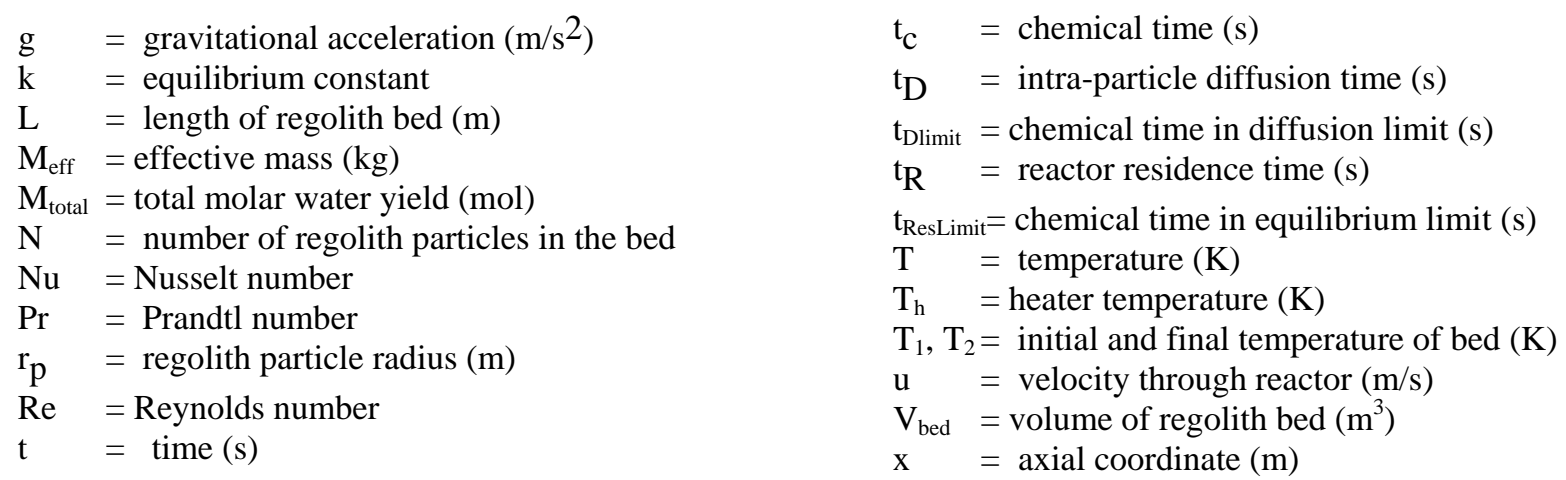

\section{ACKNOWLEDGMENTS}

U. Hegde and R. Balasubramaniam were supported under NASA Cooperative Agreement NCC3-975 with the National Center for Space Exploration Research. The authors greatly appreciate the encouragement and insights offered by K. Sacksteder and D. Linne of NASA Glenn Research Center, and useful discussions with E. SantiagoMaldonado of NASA Kennedy Space Center during the course of this work.

\section{REFERENCES}

Borodulya, V. A., Teplitsky, Y. S., Markevich, I. I., Hassan, A. F., and Yeryomenko, T. P., "Heat Transfer Between a Surface and a Fluidized Bed: Consideration of Pressure and Temperature Effects," International Journal of Heat and Mass Transfer, 34,47-53, (1991).

Chambers, J. G.,"Quantitative Mineralogical Characterization of Lunar High-Ti Mare Basalts and Soils for Oxygen Production,” Journal of Geophysical Research, 100, 14391-14401, (1995).

Hegde, U., Balasubramaniam, R., and Gokoglu, S., Development of a Reactor Model for Chemical Conversion of Lunar Regolith, Space Technologies and Applications International Forum, Albuquerque, NM, 2007.

Heiken, G. H. Vaniman, D. T., and French, B. M., Lunar Sourcebook, Cambridge University Press, New York, NY, 1991, pp. 285-300.

Levenspiel, O.,ChemicalReaction Engineering, John Wiley \& Sons, New York, NY, 1998, pp. 424-498.

McKay, D. S., and Allen, C. C., Hydrogen Reduction of Lunar Materials for Oxygen Extraction on the Moon, AIAA Paper No. 96-0488, American Institute of Aeronautics and Astronautics, Washington, DC, 1996.

Rice, E.E., Hermes, P. A., and Musbah, O. A., Carbon Based Reduction of Lunar Oxides for Oxygen Production, AIAA Paper No. 97-0890, American Institute of Aeronautics and Astronautics, Washington, DC, 1997.

Santiago-Maldonado, E., ISRU SystemModel Tool: From Excavation to Oxygen Production, Space Resources Roundtable Inc., Golden, CO, 2007.

Taylor, L. A., and Carrier, W. D. III, "Production of Oxygen on the Moon: Which Processes are Best and Why,” AIAA Journal, 30, (12), 2858-2863, (1992).

Williams, R. J., and Erstfeld, T. E., High Temperature Electrolyte Recovery of Oxygen from Gaseous Effluents from the Carbochlorination of Lunar Anthracite and the Hydrogenation of Ilmenite: A Theoretical Study, NASA TM 58214, NASA, Washington, DC, 1979.

Yoshida, H., Watanabe, T., Kanamon, H., Yoshida, T., Ogiwara, S., and Eguchi, K., Experimental Study on Water Production by Hydrogen Reduction of Lunar Soil Simulant in a Fixed-Bed Reactor, Space Resources Roundtable Inc., Golden, CO, 2000. 